\title{
Métodos de homogenización y bifurcaciones en modelos sobre dispersión ecológica
}

\author{
Homogenization methods and bifurcations \\ in models on ecological dispersion
}

\author{
Mauro Montealegre Cardenas \\ mmonteal@usco.edu.co \\ Jasmidt Vera Cuenca \\ jasmidt.vera@usco.edu.co \\ Edgar Montealegre Cardenas \\ ingemco@hotmail.com
}

\begin{abstract}
Resumen
Este artculo sobre metodos de la modelamiento para sistemas ecologicos resulto del proyecto de investigacion denominado $\backslash$ Movilizacion Matematica y Sistemas Dinamicos en algunos Ecosistemas $\backslash$, donde estudiamos el paradigma de los sistemas dinamicos para la descripcion de la dispersion ecologica. Este enfoque ha sido muy exitoso para conocer variedad de fenomenos de las ciencias naturales y sociales; pero debido a las interacciones complejas entre: clima, suelo, agua, biotica, la presencia de la memoria en los procesos dada por la historia local integrada a un entorno, a la morfo-dinamica moldeada por el ujo de poblaciones, resulta que los ecosistemas son mas complejos y deben ser considerados como un sistema dinamico adaptativo o emergente. En este trabajo escribimos la evolucion de ecosistemas, que se caracterizan por : interdenicion de sus variables a partir de la toma de datos, la estimacion estadstica de los parametros, la existencia de varias escalas espaciotemporales; por ello integramos metodos estadsticos con los cambios de variables multiescala para homogenizar y promediar sus perturbaciones singulares.
\end{abstract}

Los modelos estudiados se aplican a procesos de migracion dispersivos que incluyen interrelaciones complejas, en donde se consideran el autocontrol resiliente, siempre y cuando las uctuaciones no superen un umbral especco.

Palabras clave: Sistemas dinamicos adaptativos, perturbaciones singulares, dispersion ambiental, homogenizacion, sistemas promediados, complejidad ambiental.

\section{Abstract}

This article on methods for ecological systems modeling research project was called "Mobilization Mathematics and Dynamical Systems in some Ecosistemas", where we study the dynamical systems paradigm for describing organic dispersion. This approach has been very successful to meet variety of phenomena in the natural and social sciences; but because of the complex interactions between: weather, soil, water, biota, the presence of memory in the process given in local history integrated into a setting, molded by the own of population-dynamics morph, is that ecosystems They are more complex and should be considered as a dynamic adaptive or emergent system.

In this work we wrote the evolution of ecosystems, which are characterized by: interdenition of its variables from the data collection, the statistical parameter estimation, the existence of multiple spatial and temporal scales; therefore we integrate statistical methods with changes multiscale variables to homogenize and averaging their singular perturbation.

Studied models apply to dispersive migration processes involving complex Interrelationships, wherein the resilient self-considered, provided that the fluctuations do not exceed a specific threshold.

Key Words: Adaptive Dynamic System, singular perturbations, environmental

dispersla, homogenization, averaging systems, environmental complexity. 


\section{Complejidad Ambiental}

El \Pensamiento Resiliente" es un nuevo paradigma sobre la Sostenibilidad en Ciencias Ambientales, se re ere a una forma uni cada de la conceptualizaci ón y gestión de los sistemas socio- ecológicos. El objetivo de la gestion de la resiliencia es mantener la capacidad de un sistema de absorber perturbaciones generadas por humanos y la recuperacion del estado natural. Cuando un sistema cruza el umbral debido a que se maneja inadecuadamente, su capacidad de recuperación disminuye y la probabilidad de un cambio de esquema a un estado inestable se incrementa. También sabemos que ciertos aspectos de un sistema ambiental incluida: la diversidad, la jerarquía y la auto-organización, tienden a aumentar la capacidad de recuperar la estructura estable del sistema.

El acercamiento a un umbral entre estados estables alternativos en un sistema conduce a un fenómeno llamado \desaceleración crítica hacia abajo", porque los indicadores deseables del sistema est an cada vez mas ausentes. Los investigadores de estas áreas usan códigos para estudiar los fenómenos naturales, eligiendo formalmente parámetros y los sistemas dinámicos como paradigma de dichos ecosistemas, un sistema dinamico es un proceso cuyo 2 estado cambia con el tiempo " (Arrowsmith y Place, 1994).

En la fundamentaci ón de los ecosistemas los científicos de las Ciencias Ambientales distinguen entidades tales como especies, formas de vida o grupos funcionales que tienen en cuenta el impacto de los procesos externos y prev en la sustitución de elementos estructurales por otra estructura.

Los sistemas ambientales automodicables, corresponden a un paradigma alternativo a los sistemas din amicos, son sistemas cuyos componentes se basan en un conjunto abierto de diferentes tipos de componentes y que puede destruir con sus actividades económicas, sus propios componentes (Kampis, 1991). Estos ecosistemas son sistemas adaptativos que pueden automodicarse produciendo nuevas variables, por ejemplo debido a la venir y ir de los organismos, a nuevos contextos ambientales, a los que aparecen expresiones fenot picas ocultas, a la competencia evolutiva o económica. Por lo tanto, los parámetros y las variables son denibles solo a posteriori.

\section{Modelos Basicos sobre Dispersion Ambiental}

Los modelos referentes al fen omeno de dispersi on espacial, Turchin (1998), correspondientes a movimientos simples de individuos de $\mathrm{m}$ ultiples especies, se realizan en ambientes heterogeneos y tienen la siguiente ecuacion basica,

$$
\frac{\partial u}{\partial t}=B \frac{\partial^{2} u}{\partial x^{2}}+\frac{\partial^{2} \Psi(u)}{\partial x^{2}},
$$

donde $u(t ; x)$ es la densidad poblacional, $\mathrm{B}$ es el coe ciente de dispersi on, es una funci on que relaciona la tendencia hacia la agregacion, Por ejemplo

; si los individuos se propagan siguiendo en camino ya existente el modelo corresponde a la ecuaci on del tel egrafo, si consideramos saltos el modelo es integrodiferencial porque involucra la probabilidad de factores exogenos.

Si solo tenemos en cuenta la alteraci on que sufre el movimiento poblacional debido a las interacciones con un medio ambiente heterogeneo y si el movimiento del individuos es aleatorio el modelo basico es entonces el siguiente,

$$
\frac{\partial u}{\partial t}=\frac{\partial}{\partial x}\left[B(u) \frac{\partial u}{\partial x}\right]
$$

donde $\mathrm{B}(\mathrm{u})$ es una funci on dependiente variables espaciales, la cual describelas alteraciones en la dispersion. Son casos particulares los modelos de tipo Fickian,

$$
\frac{\partial u}{\partial t}=\frac{\partial}{\partial x}(B(x)) \cdot \frac{\partial}{\partial x}(u(t, x)) ;
$$

o modelos no lineales de Reaccion-Difusi on con coe cientes dependientes de variables espaciales, como el siguiente modelo Kolmogorov,

$$
\frac{\partial}{\partial t} u(x, t)=\frac{\partial}{\partial x}\left[B(u) \frac{\partial u}{\partial x}\right]+F(u) .
$$

Fischer considero el caso $F(u)=u(u-a)(u-1) \operatorname{con} 0<a<1$ :

Las interrelaciones entre consumo y conservacion de recursos naturales son fen omenos de dispersi on nohomogeneas y no-autonomas, son muy frecuentes en la denominada difusion ecologica, estos fenomenos se expresan con estas ecuaciones diferenciales parciales cuyas variables espaciales y temporales generalmente tienen escalas rapidas,

De estos modelos concretos $\mathrm{m}$ as frecuentes para describir la difusion ecolgica son los de Fokker-Planck no autonomos

$$
\frac{\partial u_{t}}{\partial t}=\frac{\partial^{2}}{\partial x^{2}}(B(x, t) u(x, t))+\frac{\partial}{\partial x^{\prime}}(v(x, t) u(x, t)) .
$$

o de Kolmogorov con variables espaciales rapidas,

$$
\frac{\partial u_{i}}{\partial t}=\operatorname{div}\left[B\left(\frac{x}{\epsilon}\right) \operatorname{grad}(u)\right]+F\left(\frac{x}{\epsilon}, u\right) \text {. }
$$

los que en el 1 mite $! 0$, probaremos respectivamente, que por procesos de auto-regresi on estad stica se convierten en los siguientes modelos homog eneos y autonomos, 


$$
\begin{aligned}
& \frac{\partial}{\partial t} c(x, t)=\left(\lim _{y \rightarrow \infty} \int_{y}^{*} \frac{1}{\delta(x, s)} d s\right)^{-1} \frac{\partial^{2}}{\partial^{2}} c(x, t), \\
& u_{t}^{0}=\operatorname{div}\left[B^{0} \operatorname{grad}^{0}\right]+\frac{1}{\operatorname{vol}(C)} \int_{C}^{*} F(y, u) d y
\end{aligned}
$$

donde B0 es constante. Finalmente para un modelo experimental de una microcuenca Lange (1994) Considero el sistema

$$
\left\{\begin{array}{l}
\frac{\partial C_{m}}{\partial t}+\left(\frac{1-\beta}{\beta}\right) \frac{\partial C_{m_{i}}}{\partial t}=B \frac{\partial^{2} C_{m}}{\partial x^{2}}-v \frac{\partial C_{m}}{\partial x} \\
\left(\frac{1-\beta}{\beta}\right) C_{m_{i}}=\alpha\left(C_{m}-C_{m_{i}}\right)
\end{array}\right.
$$

donde es el contenido de agua, es el coe ciente de transferencia.

\section{El Metodo de Escalas Multiples en Sistemas Dispersion}

En esta secci on consideramos cuatro casos sobre dispersi on singularmente perturbados, a los que le aplicamos el metodo de homogenizacion

\section{Problemas con Escalas Temporales Multiples}

Nos jamos en la siguiente ecuaci on no amortiguada con perturbaciones singulares de Dung

$$
\frac{d^{2} y}{d t^{2}}+y+\epsilon y^{3}=0 ; y(0)=1, y^{\prime}(0)=0,
$$

la no linealidad cubica es de orden $\mathrm{O}($.

Podemos pensar en una escala de tiempo \lento" ya que si es peque no, la cantidad t aumenta lentamente cuando t cambia por orden uno, por ello introducimos una variable lenta explcita $\mathrm{T}=\mathrm{t}$;

ahora expresamos su solucion tanto de $t$ como en $\mathrm{T}, \mathrm{y}=$ $\mathrm{y}(\mathrm{t} ; \mathrm{T})$; expandida para :

$$
y(t)=y_{0}(t, T)+\epsilon y_{1}(t, T)+\cdots,
$$

reemplazamos en la anterior ecuaci on de Du ng y obtenemos,

$$
\begin{gathered}
\frac{d y}{d t}=\left(\frac{\partial y_{0}}{\partial t}+\frac{d T}{d t} \frac{\partial y_{0}}{\partial T}\right)+\epsilon\left(\frac{\partial y_{1}}{\partial t}+\frac{d T}{d t} \frac{\partial y_{1}}{\partial T}\right)+O\left(\epsilon^{2}\right) \\
=\frac{\partial y_{0}}{\partial t}+\epsilon\left(\frac{\partial y_{0}}{\partial T}+\frac{\partial y_{1}}{\partial t}\right)+O\left(\epsilon^{2}\right) .
\end{gathered}
$$

Diferenciando una vez mas, obtenemos,

$$
\frac{d^{2} y}{d t^{2}}=\frac{\partial^{2} y_{0}}{\partial t^{2}}+\epsilon\left(2 \frac{\partial^{2} y_{0}}{\partial t \partial T}+\frac{\partial^{2} y_{1}}{\partial t^{2}}\right)+O\left(\epsilon^{2}\right) .
$$

Las ecuaciones diferenciales para $0 ; 1 ; 2$; son respectivamente:

$$
\begin{gathered}
\frac{\partial^{2} y_{0}}{\partial t^{2}}+y_{0}=0 \\
\frac{\partial^{2} y_{1}}{\partial t^{2}}+y_{1}=-y_{0}^{3}-2 \frac{\partial^{2} y_{0}}{\partial t^{2}} \\
\ldots
\end{gathered}
$$

La ecuaci on de orden cero del oscilador arm onico ecuacion, tiene coecientes que involucran la variable $T$,

$$
y_{0}(t . T)=A(T) e^{*+}+A^{*}(T) e^{-i t},
$$

donde $\mathrm{A}(\mathrm{T})$ es una funcion de la variable lenta $\mathrm{T}$ y por la periodicidad con respecto a $t$, y0 $=C(T) \cos (t)+$ $\mathrm{S}(\mathrm{T}) \operatorname{sen}(\mathrm{t})$ con coecientes $\mathrm{C}(\mathrm{T})$ y $\mathrm{S}(\mathrm{T})$ determinados por las condiciones iniciales. El lado derecho de la ecuacion de orden "2 se puede escribir en la forma:

$$
\begin{aligned}
-y_{0}^{3}-2 \frac{\partial^{2} y_{0}}{\partial t^{2}}=e^{i t} & \left\{-2 i \frac{d A}{d T}-3|A|^{2} A\right\} \\
& +e^{3 i t}\left\{\left|1-A^{3}\right|\right\}+c . c . .
\end{aligned}
$$

Los $t$ erminos e it son soluciones con coe cientes constantes igual a cero, por ello resulta la siguiente ecuacion,

$$
\left\{\begin{array}{l}
\frac{\partial^{2} y_{1}}{\partial t^{2}}+y_{1}=0 \\
-2 i \frac{d A}{d T}-3|A|^{2} A=0 .
\end{array}\right.
$$

Por lo tanto, podemos esperar que la ecuacion expansi on sea valida solo hasta tiempos $t<1=2$ y la solucion de la ecuacion de amplitud en la forma polar es,

$$
A(T)=a(T) e^{i \theta(T)} .
$$

resultando dos ecuaciones diferenciales ordinarias acopladas para la magnitud y la fase:

$$
\frac{d a}{d T}=0, \frac{d \theta}{d T}=\frac{3}{2} a^{2} .
$$


lo que se integra facilmente para dar

$$
\theta(T)=\theta_{0}+\frac{3}{2} a^{2} T
$$

donde 0 es otra constante de integracion. Llegamos a la conclusi on de que la soluci on de la ecuaci on de amplitud tiene la forma

$$
A(T)=a e^{i \theta}=a_{0} e x p\left[i\left(\theta_{0}+\frac{3}{2} a_{0}^{2}\right)\right],
$$

y por lo que la solucion de el orden cero la solucion de la ecuacion de Dung esta dada por

$Y_{0}(t, T)=A(t) c^{i t}+c_{c} c_{.}=2 a_{0} \cos \left(\theta_{0}+\frac{3}{2} a_{0}^{2} T+t\right)$

para determinar los valores de las constantes a 0 y 0 ; podemos asumir que

$$
\begin{gathered}
y(0)=1 \Rightarrow y_{0}(0,0)=1, y_{1}(0,0)=1 \\
y^{\prime}(0)=0 \Rightarrow \frac{\partial y_{0}}{\partial T}(0,0)=0, \frac{\partial y_{1}}{\partial T}(0,0)=-\frac{\partial y_{0}}{\partial T}(0,0)
\end{gathered}
$$

donde@tyl=..@ty0; entonces se deduce que,

$$
\left\{\begin{array}{l}
y_{0}(0,0)=2 a_{0} \cos \left(\theta_{0}\right)=1 \\
\partial y_{0}(0,0)=-2 a_{0} \operatorname{sen}\left(\theta_{0}\right)=0 .
\end{array}\right.
$$

Finalmente la solucion multiescala de orden cero para la ecuacion singular de Dung resulta ser,

$$
Y_{0}(t, T)=\cos \left[t+\frac{3}{8} T\right]=\cos \left[t\left(1+\frac{3}{8} \epsilon\right)\right] .
$$

\section{Problemas con escalas especiales múltiples}

Sea RN;N 1; T $>0$; sea una funcion espacialmente peri odica $f(t ; x) 2$ L2( $(0 ; T)$ ), a 2 L2(), consideramos variables microscopicas $\mathrm{x} 2$ y variables macroscopicas $\mathrm{y}=\mathrm{x}$ para $>0$ peque no; aplicamos un proceso de la teor a de perturbaci on multiescalar, G. Allaire (2010), para transformar el siguiente problema de Cauchy $(\mathrm{P})$ :

$P_{\epsilon}: \begin{cases}C\left(\frac{x}{\epsilon}\right) \frac{\partial u_{\epsilon}}{\partial t}-\operatorname{div}\left(B\left(\frac{x}{\epsilon} \nabla u_{\epsilon}\right)\right)=f(t, x) \\ u_{\epsilon}=0 \quad \text { en } & (0, T) * \partial \Omega \\ u_{\epsilon}(0, x)=a(x) & \text { en } \quad \Omega\end{cases}$ en un sistema homog eneo ( $\mathrm{P0}$ ); donde $\mathrm{B}$ es espacialmente peri odica y satisface la siguiente propiedad coercitiva,

$\alpha|\xi|^{2} \leq \sum_{i, j=1}^{N} B_{i, j}(y) \xi_{i} \xi_{j} \leq \beta|\xi|^{2}, \beta>\alpha \geq 0$.

Asumimos que la soluci on de $(\mathrm{P})$ tiene la siguiente expansion

$$
u_{\epsilon}(t, x)=\sum_{i=0}^{\infty} \epsilon^{i} u_{i}\left(t, x, \frac{x}{\epsilon}\right),
$$

Podemos aplicar el m etodo del doble reescalonamiento singular para obtener que: $u 0(t ; x ; y)$ $=\mathbf{u}(t ; x)$ satisface el siguiente problema homogeneo $\mathrm{P0}$ :

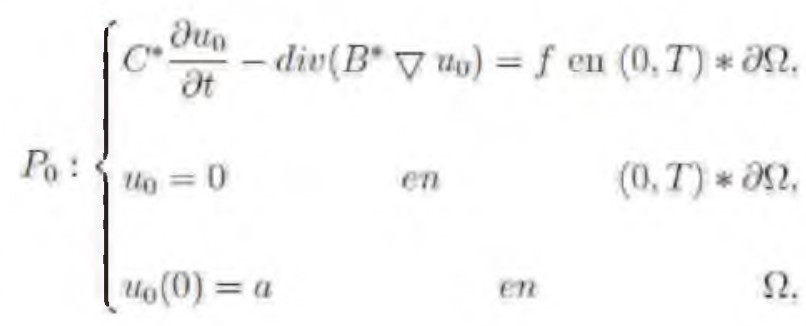

donde $\mathrm{C}=$ en Wi es solucion al siguiente problema de la celula unitaria,

$$
-\operatorname{div}(D(y) \nabla u-W(y))=f .
$$

Cuando el problema (P) es aut onomo, lo cual se consigue con un cambio de variable espacial, el sistema lmite homogeneo alcanza la siguiente forma:

$$
\begin{array}{r}
u_{i}^{0}=\operatorname{div}\left(u^{0} \operatorname{grad} u^{0}\right)+F^{0}\left(u^{0}\right), F^{0}(u) \\
=\frac{1}{\operatorname{vol}(C)} \int_{C} f(y, u) d y .
\end{array}
$$

Formas Normales para Dispersion con Reaccion Peri odica Rapida

Consideramos una version heter ogenea temporal que se encuentra en el termino de reaccion periodica, ver B. Friedler (2001),

$$
u_{t}=\Delta_{x, y} u+f\left(\frac{t}{\varepsilon}, u\right)
$$

donde reescribimos esta ecuaci on con los siguientes cambios de variables,

$$
u=\left(\begin{array}{c}
u \\
u_{x}
\end{array}\right), \quad B=\left(\begin{array}{cc}
0 & I \\
-\Delta_{y} & 0
\end{array}\right), \quad F\left(\frac{t}{\varepsilon}, u\right)=\left(\begin{array}{c}
0 \\
-f\left(\frac{t}{\varepsilon}, u\right)
\end{array}\right)
$$


Obtenemos de nuevo una ecuacion

$$
u_{t}=B u+F\left(\frac{t}{\varepsilon}, u\right)
$$

La correspondiente ecuacion de reaccion es periodica, $\mathrm{R}$; la cual convertimos en aut onoma aumentando el sistema en una dimension $2(\mathrm{RZ}) \mathrm{m}=\mathrm{Tm} ; \mathrm{m} 2 \mathrm{~N}$; para obtener

$$
\left\{\begin{array}{l}
u_{\imath}=B u+F(\phi, u) \\
\dot{\phi}=\frac{1}{\varepsilon} G(\phi, u)
\end{array} .\right.
$$

con $\mathrm{G}(; \mathrm{u}) \quad \mathrm{c} 0>0$, donde $\mathrm{u} 2 \mathrm{X}, \mathrm{X}$ alg un espacio apropiado para el operador B. Para este sistema singular u se expresa proximo de la identidad en una bola BR(X) en la siguiente forma,

$$
u=v+\epsilon W(\phi, u)
$$

esta ecuacion se puede derivar para obtener

$$
\partial_{t} u=\partial_{t} v+\varepsilon \partial_{v} W(\phi, v) \partial_{t} u+\varepsilon \partial_{\phi} W(\phi, v) \partial_{t} \dot{\phi}_{;}
$$

reemplazando en el sistema de arriba obtenemos:

$$
\begin{array}{r}
\partial_{t} v=\left(I+\varepsilon \partial_{v} W(\phi, v)\right)^{-1}\{B(v+\varepsilon W(\phi, v)) \\
+f(\phi, v+\varepsilon W(v, \phi))
\end{array}
$$

"G(v; )g: Por la teor a de Formas Normales dependiendo de $\mathrm{G}$, se puede tratar de simplicar el termino F con una eleccion apropiada de W;

$$
\begin{gathered}
F(v, \phi)=\hat{F}(v)+g(\phi, v) \text { tal que } \\
\langle g\rangle=\int_{T^{\mathrm{m}}} g(\psi, v) d v=0 .
\end{gathered}
$$

En el caso $m$ as simple de forzamiento externo peri odico, $\mathrm{m}=1$ y $\mathrm{G} 1$, hacemos,

$$
W(v, \phi)=\int_{0}^{\phi} g(\psi, v) d \psi,
$$

entonces la ecuacion transformada adquiere la forma

$$
\begin{gathered}
\partial_{t} v=\left(I+\varepsilon \partial_{v} W(\phi, v)\right)^{-1}\{B(v+\varepsilon W(\phi, v)) \\
+\hat{F}(v+\varepsilon W(\phi, v), \phi)+g(v+ \\
\epsilon W(\phi, v), \phi)-g(\phi, v)\} .
\end{gathered}
$$

Tratamiento an alogo se da al siguiente sistema de ecuaciones de reacciondifusi

$$
\left\{\begin{array}{l}
u_{t}=B \Delta u+F(u)+g(\phi, u) \\
\phi=\frac{1}{\varepsilon} G(\phi, u) \\
(\phi(0), u(0)) \in X * T^{m}
\end{array}\right.
$$

Despu es de aplicar la normalizaci on anterior, surge una cuesti on importante es la descripci on del comportamiento cerca de un equilibrio para el sistema de la forma,

$$
u_{t}=B u+F(u), u \in \mathbb{R}^{n} .
$$

cuando $\mathrm{B}$ tiene espectro puramente imaginario $\mathrm{y}$ semisimple, esto es no hay bloques de Jordan de tama no 2 o mas grande en la forma normal de Jordan compleja. La estabilidad de la solucion u0 depende de los valores propios de DuFO(u0); pues si uno de sus valores propios tiene parte real cero estamos ante la presencia de una bifurcacion de Hopf para la variable espacial.

Para el reconocimiento de patrones expresamos las soluciones del problema $\mathrm{PO}$ en terminos de frecuencias as,

$$
u_{0}(t, x)=A(\tau, x, y) Q^{i q_{c} 2 x}+t .0 . S .
$$

donde qc es el numero de onda cr tico; la ecuacion de la amplitud $A(; x ; y)$ se consigue para 1 en la sustitucion de la expansion u en (P). En este caso exp(Bt) es peri odico con periodo $\mathrm{b} \mathrm{T}$, entonces todos los t erminos resonantes pueden ser removidos por cambios apropiados de coordenadas, excepto los que sean invariantes bajo el operador de promedio temporal ;

$$
\pi F(u)=\frac{1}{T} \int_{0}^{\hat{T}} \exp (-B t) F(\operatorname{cxp}(B t) u) d t .
$$

\section{Homogenizaci on espacio-temporal para Difusi on Ecologica}

La propagacion de una poblaci on o enfermedad esta conectado a la circulacion de los individuos en habitats locales, as como en paisajes grandes podemor asumir que el coe ciente de difusi on depende de dos escalas espaciales, uno que var a r apidamente en distancias corto, por ejemplo, debido a las caracter sticas de la vegetacion y una variable correspondiente escalas 10 de paisaje, Turchin (1998) introduce un par ametro, , que es la relacion de las peque nas y grandes escalas, es decir, sea x la escala espacial grande con una asociada escala de tiempo lento, t. Entonces la variable espacial puede expresarse como $\mathrm{x}=\mathrm{y}$, mientras que el tiempo asociado esta relacionado por $\mathrm{t}=$ 2 . Murray (1989) estima que el tiempo promedio para que un organismo en viajar una distancia L es

$$
\frac{\partial L^{2}}{\partial B} \ln \left(\frac{L}{a}\right)
$$


De la seccion 4 de este artculo, suponiendo que no hay ning un componente de advecci on, obtenemos la siguiente ecuacion de difusion ecologica,

$$
\frac{\partial u}{\partial t}=\frac{\partial^{2}}{\partial x^{2}}(B(u))
$$

$\mathrm{u} u(\mathrm{x} ; \mathrm{t})$ y $\mathrm{B} \mathrm{B}(\mathrm{x} ; \mathrm{t})$ representa el coeciente de difusion que podr an variar con el tiempo y el espacio; $B$, es la motilidad de los induviduos y se supone que vara en el espacio con dos escalas.

Suponemos que la variable dependiente $\mathbf{u}$ puede escribirse como una serie de potencias en , $\mathrm{u}=\mathrm{u} 0+\mathrm{u} 1$ $+2 \mathrm{u} 2+:::$ donde es peque no, mas cercano a cero que de uno. La soluci on $\mathrm{u} 0$, se conoce como la aproximacion de primer orden de $u$. Transformamos las derivadas en terminos de las nuevas variables $\mathrm{x}$; $\mathrm{y}$; $\mathrm{t}$; ; y de la sustituci on de u con la anterior serie de potencias obtenemos,

$$
\begin{gathered}
\left(\frac{\partial}{\partial \tau}+\epsilon^{2} \frac{\partial}{\partial t}\right)\left(u_{0}+\epsilon u_{1}+\epsilon^{2} u_{2}+\ldots\right) \\
=\left(\frac{\partial^{2}}{\partial y^{2}}+2 \epsilon \frac{\partial^{2}}{\partial x \partial y}+\epsilon^{2} \frac{\partial^{2}}{\partial x^{2}}\right) B(x, y)\left(u_{0}+\epsilon u_{1}+\epsilon^{2} u_{2}+\ldots\right)
\end{gathered}
$$

Esta ecuaci on se puede dividir en una serie de ecuaciones simples igual andolas segun las potencias de . Comenzamos por reunir las condiciones de primer orden, 0, para formar la ecuacion @u0@=@2@y2 (B(x; y)u0). Tengamos en cuenta que esta es una ecuacion de difusi on con escala espacial peque na, y, escala de tiempo $\mathbf{r}$ apido, . Por lo tanto el equilibrio de esta ecuacion es@2 Tambien la ecuacion formada por el termino con $1, \mathrm{u} 1=\mathrm{b}(\mathrm{x} ; \mathrm{t})=\mathrm{B}(\mathrm{x} ; \mathrm{y})$. La ecuacion para 2 es la siguiente,

$$
\frac{\partial u_{2}}{\partial \tau}+\frac{\partial u_{0}}{\partial t}=\frac{\partial^{2}}{\partial y^{2}}\left(B(x, y) u_{2}\right)+\frac{\partial^{2}}{\partial x^{2}} B\left(\delta(x, y) u_{0}\right) .
$$

Como en las ecuaciones para 0 y 1 , hay una derivada de la escala de tiempo rapida , por lo que la solucion a esta ecuacion se aproxima $r$ apidamente a la soluci on de la ecuacion de estado estacionario con respecto a, . La escala de tiempo es, la ecuacion se convierte en,

$$
\frac{\partial^{2}}{\partial y^{2}}\left(B(x, y) u_{2}\right)=\frac{\partial}{\partial t}\left(\frac{c(x, t)}{B(x, y)}\right)-\frac{\partial^{2}}{\partial x^{2}} c(x, t) .
$$

Puesto que la derivada parcial con respecto a y es cero, resulta que $\mathrm{ul}=0$, as que c; es constante en y y R Y representa el subconjunto de soporte espacial sobre el cual se desea promediado. Los dos ultimos terminos del lado derecho crecen $\sin 1$ mites cuando y tiende hacia el in nito. Este comportamiento no ocurrir a en una soluci on $\mathrm{v}$ alida, por lo que una condici on necesaria es que la suma de estos dos terminos es igual a cero, es decir, $\lim _{y \rightarrow \infty}\left[\frac{\partial}{\partial t} c(x, t) \int_{Y} \frac{1}{B(x, s)} \partial s-y \frac{\partial^{2}}{\partial x^{2}} c(x, t)\right]=0$

Esta condicion con algunas produce la siguiente ecuaci on de difusi on homogeneizada, El coeciente de difusi on $\mathrm{B}$; es la media arm onica de B sobre la escala pequena.

\section{Detección de Formación de patrones en Sistemas Dispersivos}

\section{Un Modelo con Dispersion Discontinua}

En el libro de Murray (2002), se propone el siguiente sistema:

$$
\frac{\partial u}{\partial t}=D_{u} \frac{\partial^{2} u}{\partial x^{2}}+f(u, v) ; \frac{\partial v}{\partial t}=\frac{\partial}{\partial x}\left(D(x) \frac{\partial v}{\partial x}+g(u, v)\right.
$$

donde f y g tienen un estado estacionario (u0; v0) distintos del origen. Por simplicidad, consideramos estas ecuaciones en dominio nito, por ejemplo $[0 ; 1]$, con condiciones de contorno cero ujo y donde,

$$
D(x)=\left\{\begin{array}{ll}
D^{-}, & 0 \leq x \leq \xi \\
D^{+}, & \xi \leq x \leq 1
\end{array} \quad \text { con } \bar{D}<D^{+} .\right.
$$

Linealizamos el sistema alrededor del estado estacionario $(\mathrm{u} 0 ; \mathrm{v} 0)$, de tal forma

que $u$.. u0 = etXu(x); v .. v0 = etXv(x): Sustituimos en el modelo linealizado de ecuaciones diferenciales ordinarias, resultando ecuaciones para

$$
X_{u}^{u}+(a-\lambda) X_{u}+b X_{v}=0
$$

son parametros en el espacio de Turing consideramos estas ecuaciones en $[0 ;)$, posteriormente en (; 1$]$. En el primer caso adicionamos a la primera ecuacion $\mathrm{s..}=\mathrm{D}$.. veces la segunda ecuacion,

$$
\left(\boldsymbol{X}_{4}+s^{n} \boldsymbol{X}_{v}\right)^{\prime \prime}+\left[a-\lambda+\frac{c s^{-}}{D^{-}}\right]+\left[X_{u}+\frac{b+(d-\lambda) s^{-} / D^{-}}{a+\lambda) c s^{-} / D^{-}} X_{v}\right]=0
$$

donde s es tal que: b+(d..)s..=D..a+)cs..=D.. = s...; es una ecuacion de segundo grado des...La ecuacion anterior se convierte en una sola ecuacion en $\mathrm{Xu}+\mathrm{s} . \mathrm{j} \mathrm{Xv}$, para $j=1 ; 2$, con solucion general Cjcos $(. . j x)+\operatorname{Dj} \operatorname{sen}(. . j x)$. Aqu $\mathrm{Cj}$ y Dj sonconstantes de integracion, ... $]=[\mathrm{a} . .+\mathrm{s} .$. $\mathrm{j}=\mathrm{D} ..] 1=2, \mathrm{j}=1 ; 2$. Por lo tanto,resultan dos ecuaciones simultaneas para Xu(x) y Xv(x) en [0; ). Luegoaplicamos las condiciones de contorno de ujo cero en la frontera $\mathrm{x}$ $=0$ yobtenemos,

$$
\begin{gathered}
X_{v}(x)=\frac{1}{\left(s_{2}^{-}-s_{1}^{-}\right)}\left[\frac{\left(\Gamma_{v}+s_{1} \Gamma_{v}\right) s_{1}^{-}}{\cos \left(\xi \alpha_{1}^{-}\right)} \cos \left(\alpha_{1}^{-} x\right)-\frac{\left(\Gamma_{u}+s_{1} \Gamma_{v}\right) s_{1}^{-}}{\cos \left(\xi \alpha_{1}^{-}\right)} \cos \left(\alpha_{2}^{-} x\right)\right] \\
X_{v}(x)=\frac{1}{\left(s_{2}^{-}-s_{1}^{-}\right)}\left[\frac{\left(\Gamma_{v}+s_{2}^{-} \Gamma_{v}\right)}{\cos \left(\xi \alpha_{2}^{-}\right)} \cos \left(\alpha_{2}^{-} x\right)-\frac{\left(\Gamma_{u}+s_{1}^{-} \Gamma_{n}\right)}{\cos \left(\xi \alpha_{1}^{-}\right)} \cos \left(\alpha_{1}^{-} x\right)\right]
\end{gathered}
$$


donde $. . \mathrm{u}=\mathrm{Xu}(), . . \mathrm{v}=\mathrm{Xv}()$. Estamos suponiendo que $\mathrm{s..}$ $16=s . .2$, u y vpueden no tener, en general, continuidad de ujo en $\mathrm{x}=$. Las solucionesde la relacion de dispersi on satisfacen $\mathrm{j}$ para algun $\mathrm{n} 2[1 ; 2 ; 3 ;:::), \mathrm{j}=1$ oj $=2$; = $1=2$ es la media de los valores propios, los cuales no son races, ya que $\cos (n=2)=0$; cuando $n$ es par.

\section{Analisis no Lineal para Formacion de Patrones Dispersivos}

Estudiaremos el siguiente sistema generico de (RDE, C. Holmes and Others, 2012)

$$
\begin{aligned}
& \frac{\partial u}{\partial t}(x, t)=f(u, v ; p)+D_{u} u_{x x}, \\
& \frac{\partial v}{\partial t}(x, t)=f(u, v ; p)+D_{v} v_{x x} .
\end{aligned}
$$

donde $\mathrm{u}$ y $\mathrm{v}$ son vectores con variables de difusi on lenta o r apida, respectivamente;Du, Dv son matrices diagonales de los coecientes de difusion; $\mathrm{p} 13$

es un vector de parametros de la reaccion; asumimos que cuando la difusionde u (resp. v) es sucientemente lenta (resp. $r$ apida) la regi on perturbadaevolucionan de acuerdo con el siguiente EDO de ecuaciones de reaccion,

$$
\begin{aligned}
& \frac{d u^{g}}{d t}(x, t)=f\left(u^{g}, v^{g} ; p\right), \\
& \frac{d v^{g}}{d t}(x, t)=g\left(u^{g}, v^{g} ; p\right), \\
& \frac{d u^{t}}{d t}(x, t)=f\left(u^{l}, v^{g} ; p\right) ;
\end{aligned}
$$

Las variables (ug; vg) representan concentraciones Iglobales" lejos de una perturbaci on especial y ul la concentracion en una region localmente perturbada.

Consideramos esta ecuacion en el intervalo $[. .1 ; 1]$, sin condiciones de ujoen la frontera $\mathrm{u}, \mathrm{v}$ en RM y u en RN, respectivamente. Para simplicar lanotacion, asumimos

$$
D_{u}=\epsilon^{2} \mathbb{I}, \quad D_{v}=D \mathbb{I},
$$

donde I es la matriz identidad de tama no adecuado. El supuesto central esque hay tres difusivilidades cin etica, lenta y rapida, segun sean 2 l D,de manera que f; $g \quad O(1)$; supongamos tambien que este sistema tiene unestado de reposo homog eneo (us(p); vs(p)) que satisface $\mathrm{f}(\mathrm{us}(\mathrm{p}) ; \mathrm{vs}(\mathrm{p}) ; \mathrm{p})=0=\mathrm{g}(\mathrm{us}(\mathrm{p}) ; \mathrm{vs}(\mathrm{p}) ; \mathrm{p})$. Consideramos condiciones iniciales de la forma

$$
\begin{array}{lll}
u(x, 0)=u^{s}, & v(x, 0)=v^{s}, & \text { en }|x|>\sqrt{\epsilon} . \\
u(x, 0)=u^{p}, & v(x, 0)=v^{p}, & \text { en }|x|<\sqrt{\epsilon} .
\end{array}
$$

donde (up; vp) es O(1) con respecto a y D. Denotamos $\mathrm{Rl}$ la region localjxj $<\mathrm{p}$ y $\mathrm{Rg}$ la region global $\mathrm{jxj}>$

Para describir la evoluci on de (u; v) en estas regiones hay que considerardiferentes escalas temporales y espaciales. Un argumento de diferentes escalasde tiempo se aplica para analizar el papel de los efectos de la reacciony difusion, se aplica una tecnica de capa 1 mite para separar las escalas espaciales.

La reacci on lenta y difusion $r$ apida inherentes a esta clase de RDE puedenser descrito para tiempos $\mathrm{t}=\mathrm{O}(1)$, $\mathrm{tu}=2 \mathrm{t}, \mathrm{y} \mathrm{tv}=\mathrm{Dt}$. Supongamosque $\mathrm{u}=\mathrm{U}(\mathrm{x} ; \mathrm{t} ; \mathrm{tu} ; \mathrm{tv}), \mathrm{v}=\mathrm{V}$ $(\mathrm{x} ; \mathrm{t}$; tu; tv). Se espera que las capas l miteest an separados en escala de longitud $O($ ), para ello escojemos coordenadas $14=(\mathrm{x} . . \mathrm{xcapa})=;$ obteniendo as dos sistemas, el primero para la regionperturbada,

$$
\frac{\partial u}{\partial t}=f+\epsilon^{2} u_{x x}, \quad \frac{\partial v}{\partial t}=g+D v_{x x i}
$$

el segundo para las regiones exteriores lejos de capas 1 mite,

$$
\frac{\partial u}{\partial t}=f+u_{\xi \xi}, \quad \frac{\partial u}{\partial t}=g+\frac{D}{\varepsilon^{2}} v_{\xi \xi}
$$

Consideremos en primer lugar la escala de tiempo de difusi on $\mathrm{r}$ apida $\mathrm{y}$ asumimos que $\mathrm{u}, \mathrm{v}$ tienen expansiones $u=u 0+u 1, v=v 0+v 1$. Sustituyendo $t v=$ Dt; primer sistema obtenemos los siguientes terminos de primer orden

$$
\frac{\partial u^{0}}{\partial t_{v}}=0, \quad \frac{\partial v^{0}}{\partial t_{v}}=v_{x x}^{0}
$$

esta claro que en las regiones exteriores, $\mathrm{u} 0=\mathrm{u} 0(\mathrm{x} ; \mathrm{t}$; tu) no evoluciona y v0evoluciona debido a la difusion de acuerdo a la siguiente expansion,

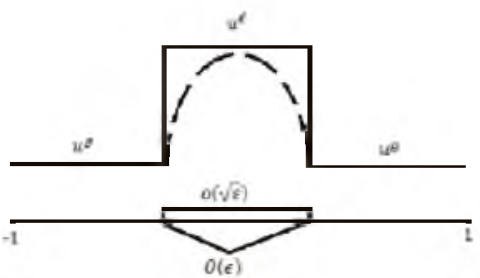

Figura 1: Efecto en la Evolucion de la Perturbacion en al Frontera

$$
\begin{aligned}
v^{0}\left(x, t, t_{u}, t_{x}\right) & =v^{0}\left(t, t_{v}\right)+\sum_{n=1}^{\infty} v^{n}\left(t, t_{u}\right) \\
& \exp \left(-(n \pi)^{2} t_{v}\right) \cos (n \pi x) .
\end{aligned}
$$


Como se ilustra que en cada regi on externa $\mathrm{Rl} ; \mathrm{g}$, v0 evoluciona a un valor constante de manera exponencial $r$ apidamente con tv.Por lo tanto (u0; v0) evolucionar a a un per l constante a trozos con diferentes valores en Rg y Rl. Luego consideremos las capas lmite entre estas regiones con escala de tiempo $\mathrm{r}$ apido. Dada la simetr a espacial, considerar solamente la capa I mite izquierda y sustituimos $t v=D t$; en el segundo sistema resulta $\mathrm{v} 0=0$, entonces

$$
v^{4}=a_{0}\left(t_{w}\right) \xi+a_{1}\left(t_{t}\right)
$$

las condiciones de frontera conducen $\mathrm{a}$,

$$
\lim _{\xi \rightarrow \infty} u^{v}(\xi)=v^{t} \quad \lim _{\xi \rightarrow-\infty} v^{0}(\xi)=v^{9}
$$

lo que implica que $\mathrm{a} 0=0$ : Cuando $\mathrm{t}=0(1)$; las soluciones se acercan a las regiones externas (ug; vg; ul)con valores constantes a trozos en Rl;g. La evoluci on de estos valores con esta escala de tiempo son descritos por la siguiente ecuacion con $t=O(1)$,

$$
\frac{\partial u^{0}}{\partial t}=f\left(u^{0}, v^{0} ; p\right) . \quad \frac{\partial v^{0}}{\partial t}=g\left(u^{0}, v^{0} ; p\right) .
$$

Sustituyendo (ug; vg) y (ul; vg), respectivamente, se obtiene las ecuaciones de reacci on mencionados arriba. Integrando la segunda de estas ultimas ecuaciones obtenemos:

$$
\begin{aligned}
\frac{\partial v^{g}}{\partial t}= & \frac{1}{2} \int_{-1}^{1} g\left(u^{0}, v^{g} ; p\right) d x=g\left(u^{g}, v^{g} ; p\right) \\
& +\sqrt{\epsilon}\left[g\left(u^{l}, v^{g} ; p\right)-g\left(u^{g}, v^{g} ; p\right)\right] .
\end{aligned}
$$

\section{Medidas Estadísticas de la Complejidad Ambiental}

Los sistemas din amicos ambientales tienen complejidades particulares, pues en estos modelos las variables corresponden a datos que deben ser tratados estad sticamente, con procesos de ltracion entre las entradas y salidas para garantizar que no pierden su naturaleza y estructura. Los modelos b asico que representan sistemas ambientales son claramente no triviales, disipativos, emergentes, explicados a partir de las leyes de la termodin amica y el c alculo de probabilidades, como lo plantea I. Prigogine y F. Takens, de las escuelas de sistemas din amicos de $B$ elgica y Holanda [Marsdeu and Schuele, 1988].

La interpretaci on de los datos estad sticos $\mathrm{x}$ (ti) correspondientes a los fen omenos de difusi on ecol ogica involucran los siguientes medidas estadsticas: a) Las sumas parciales, $y[w]=\sum_{k=1}^{n} x\left(t_{n}\right)$

b) Desviacion con respecto de un incremento lineal de las sumas parciales en un rango dado de tiempo $\mathrm{k}$,

$$
D(n, i, k)=y(n+i)-y(n)-\frac{i}{k}(y(n+k)-y(n)
$$

c) Rango estadstico, R(n; k) = Dmaxx0

d) Desviaciones $S(n ; k)=$

e) Indice estadstico, q(n; k) = R(n;k)

f) $\mathrm{Si}$ los datos $\mathrm{X}$ se expresan en un alfabeto $\mathrm{A}$ con palabras de longitud $L, x i$ ! ai; $A=$ aj $\operatorname{con} j=0 ; 1 ; 2 ; \ldots ; A$.. $1 ;$. Las p erdidas de informaciones son dada por la entropa de Shannon, HL, donde

$$
H_{L}=-\sum_{p_{N}}^{L} \ln \left(p_{i}^{L}\right)
$$

g) La ganancia de informacion es $M I G=H L$.. HL.. 1;

h) La complejidad de las uctuaciones es dada por 2 $\mathrm{k}=\mathrm{Pi} ; \mathrm{j} \mathrm{pi} ; \mathrm{j}(\ln (\mathrm{pipj})) 2$; donde pi y pij son las frecuencias $\mathrm{y}$ frecuencias relativas para que tal smbolo aparezca en esas palabras de longitud L:

\section{Estimacion de Parametro y Trayectorias}

En los modelos de dispersion ecologica se involucran relaciones socio-ambientales, por ello sus soluciones sostenibles deseadas involucran variables ambientales y economicas, tal como se ilustra en la siguiente gura

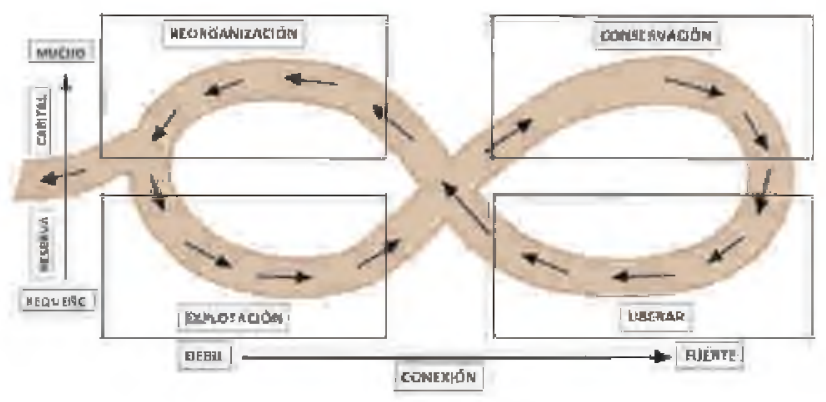

Figura 2: Periodisidad en un Sistema Ambiental Complejo

Por ello para el conocimiento de par ametro y soluciones de modelos dispersivos recurrimos a procesos estoc asticos porque son los que mejor se aproxima al mundo real. 


\section{Los Datos y sus mediciones}

Podemos representar el sistema que representa la dispersion que puede escribirse a traves de la siguiente E.D.O.,

$$
w(t)=f(2(t), \lambda)
$$

donde el vector de parametros contiene todos los par ametros ambientales y de medici on. Para estos fen omenos los estados $\mathrm{x}(\mathrm{t})$ no pueden ser medidos directamente, lo que observamos son cantidades $y(t)$ que se relaciona con $\mathrm{x}(\mathrm{t})$ por una transformacion dada por una funci on de medici on suave que algunos errores aleatorios independientes (t); las mediciones se realizan con un intervalo de muestreo jo $t \mathrm{t}$; $\mathrm{t}+\mathrm{t}$; :.:; $\mathrm{t}+(\mathrm{N} . .1$ ) $\mathrm{t}$ : Se supone de una funcion de medicion $\mathrm{G}$ : RDx ...! RDy que asigna el estado vector $\mathrm{x}(\mathrm{t})$ a una cantidad de dimension menor o igual y(t); y los errores de medicion $t$ se asumen Gaussianos con media cero, entonces la ecuacion de medida se convierte en,

$$
y_{t}=G\left(x_{t}, \lambda\right)+\eta_{t},
$$

produciendo una serie temporal multivariante yti $(\mathrm{i}=1$; :::;N). Este modelo estoc astico sobre dispersi on ambiental que se encontro (J. Timmer, 2002) se pueden formular as:

$$
\left\{\begin{array}{l}
x_{t+\Delta t}=F\left(x_{t}, \lambda\right) \\
y_{t+\Delta t}=G\left(x_{t+\Delta x}, \lambda\right)+\eta_{t+\Delta t}
\end{array}\right.
$$

El paso de tiempo $t$ corresponde al tiempo de muestreo, la funcion $F$ se obtiene de la funcion $f$ en la ecuacion de dispersion a traves del operador

$$
F\left(x_{t}, \lambda\right)=x_{t}+\int_{t}^{t+\lambda t} f(x(T), \lambda) d T
$$

Los estados descritos obedecen a un proceso de Markov, es decir, cada estado sigue unicamente de su predecesor. Un modelo lineal tiene la siguiente forma

$$
x_{t+\Delta t}=F(\lambda) x_{l}+\varepsilon_{t}: H_{t+\Delta t}=G(\lambda) x_{t+\Delta t}+\eta_{t+\Delta t}
$$

Las covarianzas del ruido del proceso y proceso de medicion se denotan respectivamente por Qty Rt,

$$
\left\{\begin{array}{l}
Q_{t}:=E\left[\tilde{E}_{t} \varepsilon_{t}^{\prime}\right] y \\
R_{t}:=E\left[\eta_{t} \eta_{t}^{\prime}\right]
\end{array}\right.
$$

donde $E[]$ es el valor esperado. Por lo tanto, "t $\mathrm{N}(0 ; \mathrm{Qt})$. Por ejemplo consideramos el caso (Timmer, 1998) de la funcion de medicion es Gxt $=$ xt 18 y las variaciones Qy R son constantes,

$$
\left\{\begin{array}{l}
x_{t+\Delta t}=F x_{t}+\varepsilon_{t,}(|F|<1) \\
y_{1+\Delta r}=x_{t+\Delta t}+\eta_{t+\Delta t}
\end{array}\right.
$$

Entonces la constante $F$ puede estimarse multiplicando ambos lados de la primera ecuacion por xt; promediando obtenemos,

$$
\hat{F}=\frac{\sum_{t} x_{t+\Delta t} x_{t}}{\sum_{t} x_{t}^{2}}=\frac{\operatorname{cov}\left(x_{t+\Delta t}, x_{t}\right)}{\operatorname{var}\left(x_{t}\right)} .
$$

Esto tambien es la estimaci on de $m$ nimos cuadrados de $\mathrm{F}$ que surge de la minimizacion de la funcion costo 2(F);

$$
\hat{F}=\arg \min _{F} \chi^{2}(F), \hat{F}=\arg \min _{F} \sum_{t} \frac{\left(x_{x+\Delta t}-F x_{t}\right)^{2}}{Q} .
$$

ra consideremos un Filtro de Kalman, F.K., todos los estados estimados hasta el tiempo $t+t$ son dadas por, $\wedge x t . . t$; $\wedge x t y$ todas sus medidas, yt; $y t+t$, se denota por $\wedge x t+t j t+t$. La estimacion a posteriori en el tiempo de referencia $\mathrm{t}+\mathrm{t}$ se escribira como $\wedge \mathrm{x}:=\wedge \mathrm{xt}+\mathrm{tjt}+\mathrm{t}$ :

Entonces la estimaci on del F.K. en el estado en el tiempo $t+t$ se realiza en dos partes:

(i) Una estimacion a priori de la situacion $\sim \mathrm{x}$ antes de la observacion de la nueva medida $\mathrm{yt}+\mathrm{t}$ :

(ii) Una correcci on despu es de la observaci on de la nueva medida que es proporcional a la diferencia de la nueva medida $\mathrm{yt}+\mathrm{t}$ y el valor predicho de la nueva medida $\sim y, \wedge x t+t j t+t=\sim x t+t j t+K t+t(y t+t . . \sim y t+t j t) ~ L a$ matriz del ltro de Kalman en $t+t$ se dene mediante la siguiente relacion:

$$
\hat{x}=\hat{x}+\hat{h}(y-\hat{y})
$$

Para el caso no-lineal, la representaci on del sistema ambiental es ahora el siguiente,

$$
\left\{\begin{array}{l}
\lambda_{t+\Delta t}=\lambda_{t} \\
x_{t+\Delta t}=F\left(x_{t}, \lambda\right) \\
y_{t+\Delta t}=G\left(x_{t+\Delta t}, \lambda\right)+J_{t+\Delta t}
\end{array}\right.
$$

Por ejemplo el modelo de Lorenz

$$
\left\{\begin{array}{l}
\tilde{x}_{1}=-\lambda_{1} x_{1}+\lambda_{2} x_{2} \\
\tilde{x}_{2}=\lambda_{2} x_{2}-x_{1}-x_{1} x_{2} \\
\tilde{x}_{3}=-\lambda_{1} x_{3}+x_{1} x_{2}
\end{array}\right.
$$

se extiende hasta el sistema de estados con dimension $\mathrm{Dx}=6$; entonces el modelo completo de espacio de estados no lineal es entonces:

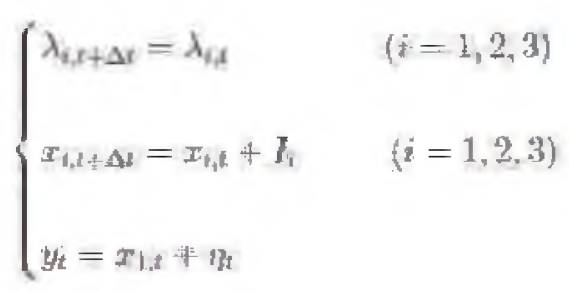




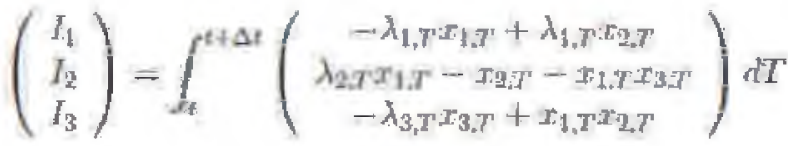

\section{Estimacion Estadstica de Parametro}

Podemos pensar en la cantidad observada de seres vivos $\mathrm{N}(\mathrm{x} ; \mathrm{t})$ y denotamos los conjuntos nitos de variables de estados y parametros as,

$$
u(t) \equiv(u(1, t), \ldots, u(x, t), \ldots, u(n, t))
$$

para $\mathrm{n}$ lugares espaciales de inter es, proceso relacionado en el siguiente modelo estad stico bayesiano,

$$
\begin{aligned}
& N(x, t) \sim[N(x, t)[u(x, t)], \forall x, t . \\
& u(x, t) \sim[u(x, t) \mid f(u(x, t), \delta)], \forall x^{1}, t,
\end{aligned}
$$

donde la notaci on [j], se re ere a una distribuci on de probabilidad condicional, representa un conjunto correspondiente a coe cientes difusi on para cadauna de las localizaciones espaciales, el modelo de difusion ecol ogica est a representadapor una aproximaci on discretizada de $\mathrm{f}(\mathrm{u}(\mathrm{t} . . \mathrm{t})$; ). Entonces,suponiendo un conjunto nito de coecientes modelados por [], dados $\mathrm{N}(\mathrm{t})$ buscamos encontrar la distribuci on condicional (es decir, a posteriori)del parametros y las variables de estado ambientales $u(t)$,

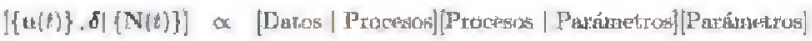

$$
\times \Pi_{l}[\mathbf{N}(t) \mid \mathbf{u}(t)] \Pi_{l}[\mathbf{u}(t) i f(\mathbf{u}(t-\Delta t), \boldsymbol{d})][\boldsymbol{d}] .
$$

donde, $\mathrm{f}(\mathrm{u}(\mathrm{t} . . \mathrm{t})$; ), se asume que contiene un conjunto de condicionesiniciales y de frontera.

Si el modelo es ajustado a un proceso de Monte Carlo, MCMC, el algoritmorequerir a la evaluaci on iterativa del modelo discretizado $f$. Ademas, amenudo hay una discrepancia entre las mediciones y la inferencia es deseada.En la situaci on espec ca con un conjunto espacialmente heterogeneo de coe-cientes de difusion (es decir, $\mathrm{B}(\mathrm{x})$ ), la dimension del espacio de parametros es muy grande.

En (Lange 1994) se una un enfoque Bayesiano para ajustar el modelo conun algoritmo MCMC, la distribuci on a posteriori correspondiente al modelo homogenizado el cual es expresado como

$$
\left.\left.\left[\left\{\mathrm{u}_{0}(t)\right] . \delta \mid[\mathrm{N}(t)]\right] \propto \prod_{t}[\mathrm{~N}(t)] \mathrm{u}_{0}(t)\right] \prod_{t}\left[\mathrm{u}_{0}(t)\right] f\left(\mathrm{u}_{0}(t-\Delta t\rangle_{i} \delta\right)\right][\delta] .
$$

Para el muestreo de los coecientes de difusion "delta.el algoritmo MCMC, la t ecnica de homogeneizaci on ayuda al maximo, en particular si usamos la razon de Metropolis-Hastings,

$$
\frac{\left.\left(\prod_{s}\left[u_{u}(t)\right) f_{h}\left(u_{n}(t-\Delta t), \delta^{(-)}\right)\right]\right)\left[\delta^{(*)}\right]\left[\delta^{(k-1)}\left[\delta^{(*)}\right]\right.}{\left.\left(\prod_{t}\left[u_{0}(t)\right] f_{h}\left(u_{0}(t-\Delta t), \delta^{(k-1)}\right)\right]\right)\left[\delta^{k-1}\right]\left[\delta^{(-)} \mid \delta^{(k-1)}\right]}
$$

En cada caso necesitamos calcular MCMC, donde K..1 es la ultima muestra MCMC para y se supone que $u 0(t)$ representa la muestra actual MCMC para el proceso homogeneizado de EDP, para el valor propuesto $\mathrm{g}(\mathrm{x})$.

La homogeneizaci on tratada en la secci on 5 de este trabajo sugiere un cambioestad stico optimo de apoyo en el proceso din amico ambiental, $y$, aunque $\mathrm{s}$ oloestamos agobiados con calcular u0(X; t), el modelo estadstico proporcionainferencia sobre basada en $u(x$; t).

En particular la dispersion de los seres vivos podemos asumir en distribucion de Poisson,

$$
\begin{aligned}
& \mathbf{N}(t) \sim \operatorname{Pois}\left(f\left(\mathbf{u}\left(t-\Delta_{l}\right), \delta(\mathrm{d})\right)\right), \forall t \\
& \mathbf{N}(t) \sim \operatorname{Poje}\left(f_{h}\left(\mathrm{u}_{13}\left(t-\Delta_{t}\right), \bar{\delta}(\mathrm{d})\right)\right), \forall t
\end{aligned}
$$

donde $f$ y fh corresponden a las soluciones de EDP originales y homogeneizadas,respectivamente. Para el estado inicial (es decir, $\mathrm{u}(0)$ ) y sin ujode condiciones de contorno, entonces buscamos la distribuci on a posterioride los coecientes de difusion, [dj fN(t); $8 \mathrm{tg}$ ].

\section{Conclusiones}

En este trabajo encontramos que para abordar matem aticamente fen omenos con estructura ambiental debemos usar intensivamente el $\mathrm{m}$ etodo de las perturbaciones singulares para homogenizar las ecuaciones de dispersi on ecol ogicas y simultaneamente usar metodos de promediacion para convertirlas en autonomas. Una vez aplicados los dos m etodos anteriores, surgen ecuaciones de amplitud, cuyas soluciones son las envolventes del sistema original y con los cuales podemos describir el fen omeno a largo plazo a trav es de las bifurcaciones globales y la identi caci on de patrones. En particular resaltamos las ecuaciones diferenciales parciales ecol ogicas, del tipo Fokker-Planck o Kolmorogov, donde la estimaci on de sus par ametros, y sus soluciones se obtienen a trav es de los procesos estad sticos que se estiman traves de la recoleccion cuidadosa de datos, los cuales se someten a Itrados para garantizar la no perdida de su naturaleza.

El sistema din amico ambiental que resulta es del tipo no Fickian, $y$ sus par ametros se estiman $v$ a correlaciones, procesos de Markov o de Monte Carlo. 
Estas interrelaciones entre determinismo y aleatoriedad se fundamentan en la naturaleza disipativa y estructurada de los sistemas ambientales, como lo reconoci o I. Prigogine, porque los conceptos de entrop a termodin amica son fundamentales para comprender su complejidad ya planteada por D. Roulle y F. Takens.

La ejecuci on de este trabajo fue favorecido por el marco te orico de la Teor a de la Complejidad, en particular la complejidad ambiental, que involucra conceptos como: disipaci on, Autoregulaci on, resiliencia, aleatoridead, entrop a, complejidad y caos aleatorio.

\section{Referencias}

Arrowsmith and PlaceArrowsmith and Place, Introduction to Dynamical Systems, Springer, 1994.

B. Fiedler, and M. Vishik. Quantitative homogenization of analytic semigroups and reaction diusion equations with diophantine spatial frequencies. Adv. Dier. Equ., 6:1377\{1408, 2001.

$G$. Allaire, Introduction to homogenization theory, Ecole Polytechnique.

France, 2010.

Doelman, R.A. Gardner, and T.J. Kaper. Stability analysis of singular

patterns in the 1d gray-scott model: a matched asymptotics approach,

Physica D: Nonlinear Phenomena, 122 (1998), pp. $1\{36$

Ferreira, M. A. R., y Lee, H. K. H., y West, M.(2006), "Multiscale

and Hidden Resolution Time Series Models", Bayesian Analysis, 1, 947-

968.

Fisher, R. A.(1937), "The Wave of Advance of Advantageous Genes",

Annals of Eugenics, $7,355\{369$.

G. Iooss, and A. Mielke. Bifurcating time\{periodic solutions of

Navier\{Stokes equations in innite cylinders. J. Nonlinear Science,

$1: 107\{146,1991$.

Henning N. Voss and Jens Timuner. Nonlinear Dynamical systems identication from uncertain and indirect measurements, international

Journal of Bifurcations and Chaos, vol.14 Nro.6(2004).

Holmes, C. Joanne Wang, Tasuku Ueno, Andrew Harwell, Leah Edelstein-

Keshet, Takanari Inoue, and Andre Levchenko. Synthetic spatially graded rac activation drives cell polarization and movement, Proceedings of the

National Academy of Sciences, Early Edition (2012).

Holmes P, Lumfey J.L., Berkooz B; turbulence, coherent structures,

Dynamical Systems and Symmetry, Cambridge, 1996.

P. G erard, P. Markowich, Homogenization limits and Wigner transforms.

Comm. Pure Appl. Math., 50: 323\{379, 1997.

Hooten, M. B. y Wikle, C. K. (2007), "Shifts in the Spatio-Temporal

Growth Dynamics of Shortleaf Pine", Environmental and Ecological

Statistics, 14, 207\{227.

23

Holmes W. R. Holmes, A. E. Regimes of wave type patterning driven by refractory actin feedback: Transition from static polarization to dynamic wave behaviour, Phys Biol, 9 (2012).

Huang, K.C. Huang and N.S. Wingreen, Min-protein oscillations in

round bacteria, Physical biology, 1 (2005), p. 229.

K. Matthies. Time-averaging under fast periodic forcing of parabolic

partial di erential equations: exponential estimates. J. Di erential Equations,

$174(1): 133\{180,2001$

Kampis G., Self Modifying Systems in Biology and Cognitive Science:

A New Framework for Dynamics, information and complexity.

Pergamon Press, Oxford, 1991.

Kim A.S., Bias Monte Carlo Methods in enviromental Engineering,

University Hawaii, 2014.

Lange, Holger y otros Modelling Input-output relations in cachments, Kluwer Academic Publishers, 1994.

Logan, J. A., y Powell, J. A. White, P., Bentz, B. J., (1998), "Model

Analysis of Spatial Patterns in Mountain Pine Beetle Outbreaks",

Theoretical Population Biology, 53 (3), $236\{255$.

Marsden and J. Scheurle, Exponentially small splittings of separatrices

with applications to KAM theory and degenerate bifurcations in

Hamiltonian dynamical systems (Boulder, CO, 1987), Contemp. Math.

81: 213\{244, Amer. Math. Soc., Providence, RI, 1988.

Murray, J. D. (2002), Mathematical Biology ,(3rd ed.), New York: Springer.

Timmer, J.; Modeling noisy time Series: physiological tremor, J. Bifurcations

and Chaos 8, 1505-1506, 2002.

Turchin, P.; Qualitative Analysis of Movement; Sunderland, MA

Sinauer, Inc. Publishers, 1998.

A.M. , The chemical basis of morphogenesis, Philosophical Transactions

of the Royal Society of London. Series B, Biological Sciences, 237

(1952), pp. $37\{72$. 